\title{
Diferenças quanto à escolaridade em adultos no desempenho no teste de cancelamento dos sinos
}

\author{
Rochele Ferronato Correa da Silva \\ Neuropsicóloga Clínica \\ Caroline de Oliveira Cardoso \\ Psicóloga Clínica \\ Rochele Paz Fonseca \\ Pontifícia Universidade Católica do Rio Grande do Sul
}

\begin{abstract}
Resumo
O papel de variáveis sociodemográficas na cognição humana, em especial da escolaridade, vem sendo estudado em indivíduos saudáveis. Esta pesquisa objetivou verificar se há diferenças entre grupos de diferentes níveis educacionais no processamento atencional visual avaliado pelo Teste de Cancelamento dos Sinos. Participaram 124 adultos jovens, distribuídos em três grupos, 5-8, 9-11 e 12 ou mais anos de educação formal, emparelhados por idade. As variáveis de acurácia e tempo, assim como os resultados qualitativos de estratégias utilizadas, foram comparados entre grupos (ANCOVA One-Way e Qui-quadrado). Observou-se uma discrepância entre dados quantitativos e qualitativos. Os grupos de diferentes escolaridades não se diferenciaram entre si quanto a acurácia e tempo; houve, porém, diferenças significativas quanto à distribuição de participantes por coluna em que o primeiro sino foi cancelado e pelas estratégias de cancelamento utilizadas. Novos estudos são relevantes com grupos de diferentes idades e com populações clínicas neurológicas e/ou psiquiátricas.
\end{abstract}

Palavras-chave: teste dos sinos; escolaridade; avaliação neuropsicológica; atenção; heminegligência.

\begin{abstract}
Differences regarding education in adults in the bells test. The role of socio-demographic variables on human cognition, particularly education, has been studied in healthy individuals. This research aimed to verify if there are differences among educational groups regarding the visual attentional processing assessed by means of Bells Test. The sample was comprised of 124 young adults, divided into three groups, 5-8, 9-11 and 12 or more years of formal education, matched by age. Accuracy and time data were analyzed by oneway ANCOVA and the qualitative results on strategies were compared between groups by Chi-square. The results showed a discrepancy between the quantitative and qualitative analysis. There were no quantitative differences among educational groups regarding accuracy and time variables. However, significant differences were found in the distribution of participants by chosen column where the first bell was canceled and the used strategies of cancellation. Further studies are relevant including different age groups and clinical samples with neurological and/or psychiatric disorders.
\end{abstract}

Keywords: Bells Test; education; neuropsychological assessment; attention; hemineglect.

\section{Introdução}

Nos países latino-americanos com frequência os testes cognitivos são apenas traduzidos de outros países, estimulandose o emprego de normas internacionais para a interpretação do desempenho de pacientes do país para o qual estão sendo introduzidos. Tal prática, sem dúvida, pode comprometer a interpretação dos dados obtidos. Além de observar a importância de se ter dados normativos para a população latino-americana, ressalta-se que estes instrumentos são padronizados graças ao entendimento de quais variáveis biológicas, socioculturais e demográficas desta população podem intervir nos processamentos cognitivos examinados.
Os fatores sociodemográficos que mais têm demonstrado efeitos significativos nos processos psicológicos avaliados por instrumentos neuropsicológicos são a idade, a escolaridade, e em alguns estudos, o gênero (Cangoz, Karakoc, \& Selekler, 2009; Heaton, Grant, \& Matthews, 1986; Kudiaki \& Aslan, 2008; Nitrini et al., 2008; Rosselli \& Ardila, 2003; Tombaugh, 2004).

Como ilustração, a educação é talvez uma das variáveis sociodemográficas com maior representatividade na pesquisa da interação destes fatores com o desempenho neuropsicológico (Ostrosky-Solís, Ramírez, \& Ardila, 2004). Os estudos investigam as funções cognitivas como atenção (Rosselli, Tappen, Williams, \& Salvatierra, 2006), percepção, linguagem 
(Machado, Correia, \& Mansur, 2007) e memória de trabalho (Talarico, Caramelli, Nitrini, \& Chaves, 2007), entre outros, na qual uma ou mais destas variáveis parecem apresentar um relevante papel nos referidos processamentos cognitivos.

O papel da escolaridade não é investigado apenas em populações saudáveis. Beausoleil, Fortin, Le Blanc e Joanette (2003), em seu estudo com as tarefas nomeação oral e fluência verbal livre, evidenciaram que o fator escolaridade pode sobrepor-se ao efeito da própria lesão cerebral no processamento de fluência verbal na avaliação de uma amostra de pacientes com lesão cerebrovascular, tamanha sua importância na cognição humana. Além disso, esta variável vem sendo relatada na literatura como uma das mais preditoras da reserva cognitiva no envelhecimento humano, prevenindo ou retardando a ocorrência de quadros demenciais (Stern, 2009).

Este fator sociodemográfico é ainda mais estudado nas pesquisas de neuropsicologia dos países em desenvolvimento, na medida em que estes apresentam um número elevado de pessoas com baixa escolaridade, ou até mesmo com analfabetismo funcional, reforçando ainda mais a necessidade de testes normatizados para a caracterização cognitiva deste perfil populacional (Ostrosky-Solís et al., 2004). Mais especificamente, no Brasil a taxa de analfabetismo entre pessoas com 15 anos ou mais é de $10,4 \%$, o que corresponde a cerca de 14 milhões de brasileiros que ainda não sabem ler nem escrever (INEP, 2007).

Além do fator de quantidade de anos de educação, em geral investigada nas pesquisas sobre a relação entre este fator sociodemográfico e a cognição, a qualidade do processo educacional se mostra igualmente um assunto de relevância ainda em ascensão nos presentes estudos, visto que nem todos os sistemas de educação se mostram equilibrados (Rosselli \& Ardila, 2003). Todavia, os instrumentos aplicados em avaliações neuropsicológicas empregam dados normativos estruturados com base predominantemente nos anos de escolaridade. Ardila, Ostrosky-Solis, Rosselli, e Gomez (2000) referiram baixos escores apresentados por indivíduos analfabetos em uma avaliação neuropsicológica. Além das diferenças de oportunidades de aprendizagem referentes às habilidades que o examinador considere relevantes, deve-se analisar o fator que contribuiu para estes reduzidos escores de desempenho o fato de os indivíduos com baixa escolaridade não estarem familiarizados com a situação formal de testagem. O efeito educativo encontrado, no entanto, não é linear, mas sim correspondente a uma curva negativa em que as diferenças entre zero e três anos de educação são altamente significativas, diferenças entre três e seis anos de escolaridade são menores, entre seis e nove são mais baixas ainda.

No que tange à avaliação da atenção, inúmeros testes de cancelamento têm sido desenvolvidos ao longo dos anos, com a finalidade de avaliar a atenção sustentada e seletiva. Os autores referem-se à atenção sustentada como a habilidade de manter o mesmo nível de consciência no desempenho ao longo de um determinado período de tempo, enquanto que a atenção seletiva implicaria na seleção de um estímulo relevante na presença de outros estímulos distratores (Mitrushina, Boone, Razan, \& D’Elia, 2005).

O Teste de Cancelamento dos Sinos, um paradigma internacional conhecido como Bells Test, desenvolvido e validado inicialmente por Gauthier, Dehaut e Joanette (1989), é considerado um dos instrumentos mais sensíveis para o diagnóstico da debilitante síndrome de heminegligência visual (Nys, Stuart, \& Dijkerman, 2010; Rorden \& Karnath, 2010; Vanier et al. 1990). Este instrumento demanda o cancelamento de alvos (sinos) dentre outros distratores, e avalia, principalmente, as funções cognitivas do processo atencional predominantemente seletivo, perceptovisual e práxico, além de velocidade de processamento.

Foi validado juntamente com outros instrumentos de desempenho e de escala funcional para o exame da heminegligência da Bateria de avaliação da negligência espacial - BEN (Azouvi et al., 2006). Até onde se sabe, embora muito explorado em populações clínicas por sua acurácia diagnóstica, há apenas um estudo publicado internacionalmente com dados de desempenho no Teste de Cancelamento dos Sinos em população saudável, com fins, dentre outros objetivos, de verificar o papel do fator sociodemográfico escolaridade (Rousseaux et al., 2001). No Brasil, apesar da crescente quantidade de publicações sobre a relevância da cultura (Andrade \& Bueno, 2007; Foss, Vale, \& Speciali, 2005) e da escolaridade (Brucki \& Nitrini, 2008; Parente, Scherer, Zimmermann, \& Fonseca, 2009; Yassuda et al. 2009) no processamento cognitivo, não parece haver nenhum estudo até o momento sobre o papel da escolaridade especificamente na atenção concentrada focalizada.

Frente aos aspectos acima revisados, parece ser indubitável a importância de se considerar o papel dos anos de estudo no desempenho neuropsicológico atencional mensurado pelo Teste de Cancelamento dos Sinos, para que um padrão de desempenho em saudáveis seja uma linha de base para comparação com populações clínicas. A partir disso, podem-se evitar falsos positivos em pacientes com escolaridade baixa, e falsos negativos em pacientes com alta escolaridade. Assim sendo, torna-se necessário promover estudos que investiguem o fator anos de estudo formal, além de outras variáveis sociodemográficas qualitativas da escolarização, e sua relação com o desempenho no instrumento em pauta. Neste contexto, o presente estudo pretende verificar se há diferenças de desempenho no Teste de Cancelamento dos Sinos entre três grupos de adultos jovens distintos quanto à quantidade de anos estudados. Tendo em vista diferenças nos resultados, objetivou-se identificar quais grupos que se diferenciaram entre si quanto ao número de omissões e ao tempo de realização da tarefa (velocidade de processamento).

\section{Método}

\section{Participantes}

Participaram 124 adultos jovens saudáveis, com idades entre 19 e 39 anos. Esta amostra foi distribuída em três subgrupos conforme a variável independente escolaridade, sendo emparelhados por idade: a) Grupo 1 - 5 a 8 anos completos de educação formal, $n=40$ (baixa escolaridade); b) Grupo 2 - 9 a 11, $n=41$ (escolaridade intermediária); e c) Grupo 3 - 12 ou mais anos de escolaridade, $n=43$ (alta escolaridade).

$\mathrm{Na}$ Tabela 1 podem ser consultados dados descritivos e 
inferenciais sobre a caracterização sociodemográfica e clínica dos participantes por grupo de escolaridade. As variáveis sociodemográficas de caracterização da amostra são idade (anos), escolaridade (anos), escore socioeconômico (utilizou-se o questionário de Critério de Classificação Econômica Brasil, proposto pela Associação Brasileira de Empresas de Pesquisa - ABEP, 2008), frequência de hábitos de linguagem escrita (escore médio), e distribuição por sexo (número absoluto e percentual). As variáveis clínicas englobam escores médios no
Inventário Beck de Depressão-II (BDI-II), no Mini Exame do Estado Mental - MEEM - e os escores ponderados médios nos subtestes das Escalas Wechsler de Inteligência para Adultos WAIS-II - Vocabulário e Cubos.

De acordo com os dados expostos na Tabela 1, houve diferenças significativas entre grupos quanto às variáveis escore socioeconômico, frequência de hábitos de linguagem e escrita, escore no MEEM, e escores ponderados médios nos subtestes Vocabulário e Cubos do WAIS-III. Embora tais diferenças

Tabela 1

Caracterização da amostra por grupos educacionais

\begin{tabular}{|c|c|c|c|c|c|c|c|c|}
\hline \multirow{8}{*}{ 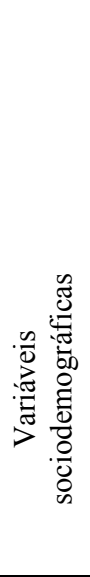 } & \multicolumn{8}{|c|}{ Grupos } \\
\hline & \multirow[t]{2}{*}{$\begin{array}{l}\text { Variáveis de } \\
\text { caracterização da Amostra }\end{array}$} & \multicolumn{2}{|c|}{$\begin{array}{c}5-8 \text { anos } \\
\text { Baixa } \\
n=40\end{array}$} & \multicolumn{2}{|c|}{$\begin{array}{c}\text { 9-11 anos } \\
\text { Intermediária } \\
\quad n=41\end{array}$} & \multicolumn{2}{|c|}{$\begin{array}{c}12 \text { ou mais } \\
\text { Alta } \\
n=43 \\
\end{array}$} & \multirow[t]{2}{*}{$p$ valor } \\
\hline & & $M$ & $D P$ & $M$ & $D P$ & $M$ & $D P$ & \\
\hline & Idade (anos) & 27,78 & 6,69 & 26,83 & 7,59 & 25,93 & 4,95 & $0,587^{\mathrm{a}}$ \\
\hline & Escolaridade (anos) & 7,00 & 1,20 & 10,78 & 0,48 & 15,57 & 2,46 & $\leq 0,001^{\mathrm{a}}$ \\
\hline & Escore socioeconômico & 20,15 & 4,94 & 26,80 & 5,05 & 30,12 & 6,78 & $\leq 0,001^{\mathrm{b}}$ \\
\hline & $\begin{array}{l}\text { Frequência de hábitos de } \\
\text { linguagem escrita }\end{array}$ & 12,65 & 5,33 & 18,66 & 4,24 & 19,70 & 4,38 & $\leq 0,001^{\mathrm{b}}$ \\
\hline & $\begin{array}{l}\text { Distribuição por sexo } \\
\text { F/M n }(\%)\end{array}$ & \multicolumn{2}{|c|}{$\begin{array}{l}25(62,5 \%) / \\
15(37,5 \%) \\
\end{array}$} & \multicolumn{2}{|c|}{$\begin{array}{c}32(78,0 \%) / \\
9(22,0 \%) \\
\end{array}$} & \multicolumn{2}{|c|}{$\begin{array}{c}24(55,1) / \\
19(44,2 \%)\end{array}$} & 0,091 \\
\hline \multirow{4}{*}{ 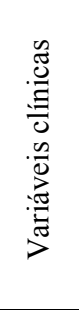 } & Escore MM & 27,38 & 1,84 & 28,46 & 1,69 & 29,29 & 0,99 & $\leq 0,001^{\mathrm{a}}$ \\
\hline & Escore BDI-II & 6,45 & 4,24 & 5,66 & 3,86 & 6,31 & 4,35 & $0,674^{\mathrm{b}}$ \\
\hline & $\begin{array}{l}\text { Escore Vocabulário } \\
\text { WAIS-III }\end{array}$ & 8,90 & 1,95 & 9,40 & 1,29 & 10,37 & 2,09 & $0,002^{\mathrm{a}}$ \\
\hline & Escore Cubos WAIS-III & 10,63 & 1,95 & 11,66 & 2,13 & 13,44 & 2,55 & $\leq 0,001^{\mathrm{a}}$ \\
\hline
\end{tabular}

pareçam ser inerentes à caracterização educacional de cada grupo, para a análise comparativa de desempenho optou-se por controlar os possíveis efeitos dos fatores socioeconômicos, e da frequência de hábitos de linguagem e escrita. Não se controlaram os efeitos das variáveis de desempenho do MEEM e dos subtestes do WAIS-III por estes serem dados de avaliação cognitiva e intelectual mais globais dos participantes para fins de caracterização, sendo esperado que possam sofrer influência de outras variáveis independentes. A amostra dos três subgrupos quanto ao gênero não foi diferente, sendo composta predominantemente por indivíduos do sexo feminino representada pela distribuição de mulheres $n=81(65,3 \%)$, e homens $n=43(34,7 \%)$.

Nos critérios de inclusão do presente estudo, os participantes foram selecionados em ambientes universitários, de trabalho e em centros de convivência, com amostragem por conveniência, ingressando apenas aqueles que apresentavam no mínimo 5 anos de educação formal; ausência de quaisquer distúrbios sensoriais (auditivos ou visuais) não-corrigidos (avaliado por auto-relato através de um questionário sócio-cultural); de sinais sugestivos de depressão (mensurados pelo BDI II, de Beck,
Steer, \& Brown,1996, adaptado ao Português Brasileiro por Finger \& Argimon, 2008) com escore abaixo de 19 pontos); de sinais sugestivos de demência (triagem feita pelo MEEM, adaptado para a população local por Chaves \& Izquierdo, 1992 , com escore $\geq 24$ ); de histórico de alcoolismo (triagem com a escala CAGE, versão utilizada no estudo de Amaral \& Malbergier, 2004; e de uso abusivo atual ou prévio de drogas ilícitas ou de benzodiazepínicos nos últimos seis meses (avaliado por auto-relato através de um questionário sócio-cultural); e de escores ponderados inferior a 7 nos subtestes Vocabulário e Cubos do WAIS-III (Nascimento, 2004). A amostra foi composta apenas por participantes brasileiros natos e falantes da língua Portuguesa, sem histórico atual ou prévio de doenças neurológicas e/ ou psiquiátricas auto-relatadas.

\section{Procedimentos}

De acordo com os aspectos éticos envolvidos nas pesquisas com seres humanos, a participação dos indivíduos no estudo foi voluntária, sendo que os instrumentos foram administrados individualmente em um ambiente apropriado. Os participantes assinaram um Termo de Consentimento Livre e Esclarecido 
do projeto de pesquisa do referido estudo aprovado pelo Comitê de Ética em Pesquisa da Pontifícia Universidade Católica do Rio Grande do Sul (CEP 09/04908). A avaliação foi realizada em uma sessão com duração aproximada de uma hora e meia, com instrumentos de caracterização da amostra e de critérios de inclusão, e com a mensuração das variáveis dependentes indicativas do desempenho atencional, perceptivo e de velocidade de processamento obtido a partir do Teste de Cancelamento dos Sinos.

\section{Instrumento}

O Teste de Cancelamento dos Sinos é um instrumento desenvolvido por Gauthier, Dehaut e Joanette (1989) e adaptado ao Português Brasileiro por Fonseca et.al. (no prelo), coordenado pela orientadora deste projeto. O teste se caracteriza pelo cancelamento de alvos (sinos) dentre distratores (outros objetos, tais como serrote, árvore, casa, maçã, peixe) que avalia, principalmente, as funções cognitivas de atenção concentrada e seletiva visual, assim como percepção visual e indiretamente velocidade de processamento e praxias para a ação de cancelar. $\mathrm{O}$ indivíduo deve cancelar todos os sinos que visualiza em uma folha onde há 315 figuras misturadas (alvos e distratores), destas 35 são sinos. A tarefa do examinando, em um primeiro momento, é localizá-los livremente, em sete colunas diferentes de conhecimento apenas do examinador. Toda vez que o indivíduo identificar um sino no meio das outras figuras deve cancelá-lo, fazendo um traço em cima do sino encontrado, o mais rápido possível. Após o participante informar o término da tarefa, o cronômetro é zerado (tempo da primeira busca) e uma folha branca é colocada em cima da folha de aplicação. Em seguida, é solicitado ao examinando verificar se traçou todos os sinos e o cronômetro é acionado novamente (tempo da segunda busca). Enquanto isso, o examinador, com uma folha gabarito, numera a ordem em que os sinos são cancelados pelo participante. Esse teste permite identificar a estratégia de busca dos sinos e se as omissões restringem-se a uma área lateralizada em particular. A pontuação inclui uma interpretação quantitativa - um escore total de omissões e erros (distratores cancelados); e o tempo de realização no primeiro e segundo momento da aplicação. Adicionalmente, há uma interpretação qualitativa da coluna em que o primeiro sino é cancelado e da estratégia de cancelamento utilizada (horizontal e vertical mista, mista, caótica).

\section{Análise dos dados}

Os dados coletados na avaliação neuropsicológica foram estudados a partir de duas análises: 1) Análise estatística descritiva, com a finalidade de caracterizar a amostra com médias, desvios-padrão de variáveis quantitativas, e frequência de ocorrência de variáveis categóricas; 2) Análise estatística inferencial de comparação de escores médios entre os grupos de escolaridade a partir do teste ANCOVA One-Way (fator escolaridade - 3 níveis), sendo o escore socioeconômico e o escore de frequência de linguagem e escrita covariantes. Fez-se uma análise com procedimentos post-hoc Scheffe. Foi utilizado o pacote estatístico SPSS versão 15.0, com um nível de significância $p \leq 0,05$.

\section{Resultados}

Os dados descritivos (média e desvio padrão) quanto aos escores do Teste de Cancelamento dos Sinos nos três subgrupos de diferentes níveis de escolaridade podem ser visualizados na Tabela 2. Na última coluna podem ser consultados os valores de $p$.

Diante desses dados, nota-se que os grupos de diferentes escolaridades não se diferenciaram entre si quanto às variáveis quantitativas de acurácia e de tempo no Teste de Cancelamento dos Sinos. Na Tabela 3 mostram-se os resultados por subgrupo quanto ao desempenho qualitativo-quantitativo no teste em estudo, mais especificamente à frequência em número absoluto e em percentual de indivíduos que começaram seu cancelamento pelas colunas $1,2,3,4,5,6$ ou 7 .

A partir dos dados, pode-se perceber que houve uma diferença significativa entre os subgrupos de baixa e de alta escolaridade quanto à frequência de cancelamento na primeira coluna $(p=0,031)$. Uma menor quantidade de participantes de baixa escolaridade cancelou seu primeiro sino na primeira coluna do que os outros subgrupos; nas colunas três e seis, em contrapartida, este mesmo subgrupo apresentou maior frequência na escolha do primeiro cancelamento quando comparado aos indivíduos de escolaridade intermediária e alta.

Em complementaridade à análise qualitativa do desempenho no Teste de Cancelamento dos Sinos iniciada na Tabela 3, na Tabela 4 pode-se observar a frequência absoluta e percentual da parcela de cada subgrupo de escolaridade que utilizou cada estratégia de cancelamento ao longo do tempo 1.

No que diz respeito à distribuição de indivíduos por grupo para cada estratégia utilizada, também evidenciou-se uma diferença significativa ( $p=0,025)$. A quantidade de participantes que utilizaram a estratégia vertical mista foi maior no grupo de 12 ou mais anos de escolaridade, sendo que a menor frequência de uso desta estratégia ocorreu no grupo de 5-8 anos de escolaridade. Além disso, os participantes de baixa escolaridade apresentaram a maior frequência de uso da estratégia caótica, em comparação aos subgrupos de escolaridade intermediária e alta, sendo que esta última teve uma frequência muito menor ao esperado no caso de uma distribuição igualitária. Mais especificamente, o grupo de 5-8 anos de estudo usou a estratégia caótica o dobro de vezes do que o esperado; em contrapartida, o grupo de $12 \mathrm{ou}$ mais anos de educação formal utilizou tal estratégia três vezes menos do que o esperado.

\section{Discussão}

O objetivo da presente pesquisa foi verificar o papel da variável sociodemográfica escolaridade no desempenho mensurado pelo Teste de Cancelamento dos Sinos. As frequentes diferenças de performance relatadas entre grupos de escolaridade em vários instrumentos neuropsicológicos denotavam encontrar diferenças quantitativas e qualitativas entre grupos de 5-8, 9-11 e 12 ou mais anos de estudo formal no desempenho no Teste de Cancelamento dos Sinos. No entanto, nos escores de omissões e na mensuração do tempo, não houve diferenças significativas 
Tabela 2

Média, desvio-padrão e nível de significância dos escores do Teste de Cancelamento dos Sinos em análise comparativa entre grupos

\begin{tabular}{|c|c|c|c|c|c|c|c|}
\hline \multirow{3}{*}{$\begin{array}{l}\text { Escores do Teste de } \\
\text { Cancelamento dos Sinos }\end{array}$} & \multirow{2}{*}{\multicolumn{2}{|c|}{$\begin{array}{c}\text { 5-8anos } \\
\text { Baixa } \\
n=40\end{array}$}} & \multicolumn{5}{|c|}{ Grupos } \\
\hline & & & \multicolumn{2}{|c|}{$\begin{array}{c}\text { 9-11 anos } \\
\text { Intermediária } \\
n=41\end{array}$} & \multicolumn{2}{|c|}{$\begin{array}{c}12 \text { ou mais } \\
\text { Alta } \\
n=43\end{array}$} & \multirow[t]{2}{*}{$p$ valor } \\
\hline & $M$ & $D P$ & $M$ & $D P$ & $M$ & $D P$ & \\
\hline Total de omissões no tempo 1 & 2,20 & 2,87 & 1,59 & 1,73 & 2,05 & 2,56 & 0,452 \\
\hline Total de omissões no tempo 2 & 0,90 & 1,69 & 0,46 & 0,75 & 0,43 & 0,80 & 0,925 \\
\hline Total tempo 1 & 119,71 & 35,74 & 105,70 & 36,70 & 95,01 & 30,58 & 0,316 \\
\hline Total tempo 2 & 48,58 & 28,13 & 50,32 & 21,54 & 56,20 & 25,31 & 0,690 \\
\hline
\end{tabular}

Tabela 3

Coluna em que o primeiro sino foi cancelado

\begin{tabular}{|c|c|c|c|c|c|c|}
\hline \multirow{3}{*}{$\begin{array}{l}\text { Coluna do primeiro } \\
\text { cancelamento }\end{array}$} & \multicolumn{6}{|c|}{ Grupos } \\
\hline & \multicolumn{2}{|c|}{$\begin{array}{c}\text { 5-8anos } \\
\text { Baixa } \\
n=40 \\
\end{array}$} & \multicolumn{2}{|c|}{$\begin{array}{c}9-11 \text { anos } \\
\text { Intermediária } \\
n=41\end{array}$} & \multicolumn{2}{|c|}{$\begin{array}{c}12 \text { ou mais } \\
\text { Alta } \\
n=43\end{array}$} \\
\hline & $n$ & $\%$ & $n$ & $\%$ & $n$ & $\%$ \\
\hline 1 & 26 & $65,0 \%$ & 36 & $87,8 \%$ & 39 & $90,7 \%$ \\
\hline 2 & 4 & $10,0 \%$ & 1 & $2,4 \%$ & 4 & $9,3 \%$ \\
\hline 3 & 2 & $5,0 \%$ & 0 & $0,0 \%$ & 0 & $0,0 \%$ \\
\hline 4 & 1 & $2,5 \%$ & 1 & $2,4 \%$ & 0 & $0,0 \%$ \\
\hline 5 & 2 & $5,0 \%$ & 0 & $0,0 \%$ & 0 & $0,0 \%$ \\
\hline 6 & 3 & $7,5 \%$ & 0 & $0,0 \%$ & 0 & $0,0 \%$ \\
\hline 7 & 2 & $5,0 \%$ & 3 & $7,3 \%$ & 0 & $0,0 \%$ \\
\hline
\end{tabular}

Tabela 4

Frequência por subgrupo de utilização das diferentes estratégicas de cancelamento

\begin{tabular}{|c|c|c|c|c|c|c|}
\hline \multirow{3}{*}{ Estratégias } & \multicolumn{6}{|c|}{ Grupos } \\
\hline & \multicolumn{2}{|c|}{$\begin{array}{c}\text { 5-8anos } \\
\text { Baixa } \\
n=40\end{array}$} & \multicolumn{2}{|c|}{$\begin{array}{c}9-11 \text { anos } \\
\text { Intermediária } \\
n=41\end{array}$} & \multicolumn{2}{|c|}{$\begin{array}{c}12 \text { ou mais } \\
\text { Alta } \\
n=43\end{array}$} \\
\hline & $n$ & $\%$ & $n$ & $\%$ & $n$ & $\%$ \\
\hline Horizontal Mista & 9 & $22,5 \%$ & 8 & $19,5 \%$ & 10 & $23,3 \%$ \\
\hline Vertical Mista & 11 & $27,5 \%$ & 15 & $36,6 \%$ & 23 & $53,5 \%$ \\
\hline Mista & 5 & $12,5 \%$ & 10 & $24,4 \%$ & 3 & $7,0 \%$ \\
\hline Caótica & 11 & $27,5 \%$ & 7 & $17,1 \%$ & 2 & $4,7 \%$ \\
\hline
\end{tabular}

quanto à variável grupo de escolaridade. Em contrapartida, foram observadas diferenças significativas interessantes na análise qualitativo-quantitativa deste instrumento. Algumas hipóteses são levantadas para explicar esta discrepância quanti-qualitativa.

Sugere-se a hipótese de facilidade do instrumento para avaliar pessoas neurologicamente saudáveis, tendo em vista que o Teste de Cancelamento dos Sinos foi desenvolvido para populações clínicas neurológicas (Gauthier et al., 1989). Outra evidência que reforça tal hipótese é a diferença encontrada entre grupos principalmente extremos de escolaridade (5-8 versus 12 ou mais anos de estudo) nos instrumentos MEEM, e subtestes do WAIS-III Vocabulário e Cubos. Na medida em que a presente amostra se diferenciou nestes três testes de avaliação cognitiva, a ausência de diferenças quantitativas entre grupos educacionais no Teste dos Sinos reflete uma grande facilidade deste paradigma para populações sem quadros neurológicos.

O levantamento da hipótese de facilidade apresenta-se, igualmente, relacionado aos resultados obtidos quanto às 
omissões tanto no primeiro quanto no segundo tempo de execução do Teste de Cancelamento dos Sinos, em que os participantes de baixa escolaridade apresentaram uma performance semelhante aos participantes de escolaridade intermediária e de nível alto, sendo que seria esperado aos indivíduos com alto nível de escolaridade mais acertos e, consequentemente, um menor número de omissões.

$\mathrm{Na}$ análise qualitativa, mesmo que o teste possa ser considerado de fácil execução, verifica-se que a amostra de adultos jovens saudáveis apresenta diferença em dois aspectos: coluna de cancelamento do primeiro sino e estratégia utilizada. O cancelamento do primeiro sino entre as pessoas de alta escolaridade ocorreu mais frequentemente na coluna de número um, sendo este cancelamento bem à esquerda da folha menos frequente entre os participantes de baixa escolaridade, o que seria considerado o ideal. Os resultados do presente estudo sugerem que o processo de educação formal pode influenciar o desempenho em tal atividade, na medida em que durante a alfabetização e a aprendizagem gradativamente mais complexa da leitura e da escrita e da própria experiência cotidiana de escolarização formal, as atividades tendem a ser conduzidas da esquerda para a direita (Byrd, Touradji, Tang, \& Manly, 2004).

Complementarmente, Azouvi et al. (2006) investigaram uma bateria de testes para avaliar a negligência unilateral e encontraram que, dentre os saudáveis, o maior número de omissões no Bells Test ocorreu entre os idosos ou aqueles com nível de escolaridade inferior. Além disso, associaram os resultados da diferença entre as omissões da esquerda e direita com a escolaridade, sendo que os de baixa escolaridade apresentaram mais omissões à esquerda, enquanto que os participantes de alta escolaridade obtiveram mais omissões à direita. Uma vez que o que torna um teste de cancelamento mais sensível para detectar heminegligência é o ponto de início do cancelamento (Nurmi et al., 2010), no Teste de Cancelamento dos Sinos a discriminância para a localização em que o primeiro sino é cancelado ocorreu, preferencialmente, entre os indivíduos de alta escolaridade à esquerda (Brucki \& Nitrini, 2008).

Adicionalmente, a maior influência da escolaridade foi observada na verificação da estratégia em que o processo de busca visual foi realizado, sendo evidenciado pelos indivíduos de alta escolaridade através de uma busca mais sistemática e organizada no cancelamento dos sinos. As estratégias previamente analisadas no estudo de Gauthier et.al. (1989) apontavam para um escaneamento mais organizado tanto na horizontal quanto na vertical em pessoas saudáveis, e os indivíduos com déficit atencional demonstravam preferência pela estratégia desorganizada e/ou caótica.

O termo qualitativo se refere a informações descritivas a respeito do comportamento apresentado pelo paciente, sendo que na avaliação qualitativa de aspectos que investigam a negligência unilateral, os clínicos observam o rendimento do participante analisando critérios que envolvem em qual lado inicia a tarefa, a estratégia de busca visual e o tempo de realização do teste de cancelamento (Plummer, Morris, \& Dunai, 2003).

Desta forma, Weintraub e Mesulam (1988) afirmaram que a estratégia de pesquisa irregular tende a estar presente quando os estímulos estão em uma matriz não estruturada, enquanto que uma busca visual estruturada solicita a organização dos estímulos de forma mais sistemática. Durante tal pesquisa os participantes que realizaram a busca de maneira aleatória foram, principalmente, os analfabetos. Estes resultados permitiram inferir que a educação formal teve uma grande influência sobre o tipo de exploração e que as pessoas analfabetas ou com mínima frequência escolar exibiram uma varredura caótica, levantando hipóteses de que o mínimo contato com elementos gráficos da escrita e da leitura, da escrita em linhas, tende a modificar a busca visual. Assim, o efeito da educação pode ser mediado tanto pela melhor estratégia de codificação ou pela melhor estratégia de reconhecimento.

Além disso, os três grupos de escolaridade foram emparelhados por idade, sendo todos adultos jovens de 19 a 39 anos de idade, sem haver a possibilidade de verificar o complexo efeito da escolaridade versus idade (Ardila et al. 2000). Talvez se a variável sociodemográfica escolaridade tivesse sido comparada entre três grupos de adultos idosos ou de adultos idosos longevos, tal diferença fosse evidenciada. Os modelos atuais retratam que o envelhecimento quando acompanhado por um estilo de vida saudável e, principalmente entre aqueles com maior nível socioeconômico e de educação, tende a contribuir e manter a eficiência cognitiva global. Assim, os anos de educação formal seriam indicadores da capacidade do cérebro em manter uma reserva cognitiva, e na habilidade de recrutamento de redes cognitivas (Stern, 2009), dados corroborados por algumas pesquisas com população clínica idosa que analisam os prejuízos no desempenho cognitivo relacionados aos níveis de escolaridade inferior (Baldivia, Andrade, \& Bueno, 2008; Katzman, 1993).

Considerando-se os resultados do presente estudo, algumas limitações podem ser levantadas. Primeiramente aponta-se restrição da pesquisa com população de adultos jovens, o que não permitiu o estudo da interação escolaridade e idade. Além disso, ocorreu a impossibilidade de emparelhar os participantes dos distintos grupos por seu desempenho no MEEM e nos subtestes da escala de inteligência na medida em que tais diferenças acompanham a característica de cada grupo.

Tendo em vista a ausência de diferenças entre grupos de diferentes escolaridades quanto aos escores quantitativos do Teste de Cancelamento dos Sinos, sugerem-se investigações que visem a relacionar a performance neste instrumento com a de outros métodos de avaliação, com o intuito de verificar se outros testes se comportam de maneira semelhante, ou seja, apresentando facilidade na execução entre indivíduos saudáveis adultos jovens. Além de outras pesquisas com testes de cancelamento, são ainda necessários estudos que analisem diferentes níveis de anos de estudo formais em diferentes grupos etários, principalmente incluindo amostras de idosos e de longevos. Sugere-se também que outros estudos procurem investigar a relação da variável sociodemográfica escolaridade em pacientes com quadros neurológicos que possam acarretar a síndrome de negligência visual unilateral.

\section{Referências}

Amaral, R., \& Malbergier, A. (2004). Avaliação de instrumento de detecção de problemas relacionados ao uso do álcool (CAGE) entre trabalhadores da 
Prefeitura do Campus da Universidade de São Paulo. Revista Brasileira de Psiquiatria, 26(3), 156-163.

Andrade, V. M., \& Bueno, O. F. A. (2007). Neuropsicologia transcultural: grupo indígena guarani. Estudos de Psicologia (Natal), 12(3), 253-258.

Ardila, A., Ostrosky-Solis, F., Rosselli, M., \& Gomez, C. (2000). Age related cognitive decline during normal aging: the complex effect of education. Archives of Clinical Neuropsychology, 15, 495-514.

Azouvi, P., Bartolomeo, P., Beis, J.M., Perennou, D., Pradat-Diehl, P., \& Rousseaux, M. (2006). A battery of tests for the quantitative assessment of unilateral neglect. Restorative Neurology and Neuroscience, 24, 273-285.

Baldivia, B., Andrade, V. M. \& Bueno, O. F. A., (2008). Contribution of education, occupation and cognitively stimulating activities to the formation of cognitive reserve. Dementia \& Neuropsychologia, 2(3), 173-182.

Beausoleil, N., Fortin, R., Le Blanc, B., \& Joanette, Y. (2003). Unconstrained oral naming performance in right and left hemisphere damaged individuals: when education overrides the lesion. Aphasiology: 17(2), 143-158.

Beck, A. T., Steer, R. A., \& Brown, G. K. (1996). BDI-II Manual. The Psychological Corporation, Harcourt Brace \& Company: San Antonio.

Brucki, S. M. D., \& Nitrini, R. (2008). Cancellation task in very low educated people. Archives of Clinical Neuropsychology, 23, 139-147.

Byrd, D. A., Touradji, P., Tang, M., \& Manly, J. J. (2004). Cancellation test performance in African American, Hispanic, and White elerly. Journal of the International Neuropsychological Society, 10, 401-411.

Cangoz, B., Karakoc, E., \& Selekler, K. (2009). Trail Making Test: normative data for Turkish elderly population by age, sex and education. Journal of the Neurological Sciences, 283, 73-78.

Chaves, M. L., \& Izquierdo, Y. (1992). Diferential diagnosis between dementia and depression: a study of efficiency increment. Acta Neurologica Scandinavia, 85, 378-382.

Finger, I. R., \& Argimon, I. I. L. (2008). Validação de constructo do Inventário de depressão de Beck - II (BDI-II) em uma população universitária. III Mostra de Pesquisa da Pós-Graduação PUCRS, Porto Alegre, Brasil.

Fonseca, R. P., Parente, M. A. M. P., Ortiz, K. Z., Soares, E. C. S., Scherer, L. C., Gauthier, L., \& Joanette, Y. (no prelo). Teste de Cancelamento dos Sinos. São Paulo: Vetor Editora.

Foss, M.P., Vale, F.A.C., \& Speciali, J.G. (2005). Influência da escolaridade na avaliação neuropsicológica de idosos. Arquivos de Neuropsiquiatria, 63(1), 119-126.

Gauthier, L., Dehaut, F., \& Joanette, Y. (1989). The Bells Test: a quantitative and qualitative test for visual neglect. International Journal of Clinical Neuropsychology, vol. XI, 2.

Heaton, R. K., Grant, I., \& Matthews, C. G. (1986). Differences in neuropsychological test performance associated with age, education, and sex. In I. Grant \& K. M. Adams (Orgs.), Neuropsychological assessment of neuropsychiatric disorders. Nova Iorque: Oxford University Press.

INEP - Instituto Nacional de Estudos e Pesquisas Educacionais Anísio Teixeira. (2007). Estudo detalha situação do analfabetismo no País. Recuperado de http://www.inep.gov.br/imprensa/noticias

Katzman, R. (1993). Education and the prevalence of dementia and Alzheimer's disease. Neurology, 43, 13-20.

Kudiaki, C., \& Aslan, A. (2008). Executive functions in a Turkish sample: sssociations with demographic variables and normative data. Applied Neuropsychology, 15(3), 194- 204.

Machado, O., Correia, S. M., \& Mansur, L. L. (2007). Desempenho de adultos brasileiros normais na prova semântica: efeito da escolaridade. Pró-Fono
Revista de Atualização Científica, 19(3), 289-294.

Mitrushina, M., Boone, K.B., Razan, J., \& D’Elia, L. F. (2005). Handbook of normative data for neuropsychological assessment. Nova Iorque: Oxford University Press.

Nascimento, E. (2004). WAIS-III: Escala de Inteligência Wechsler para Adultos. São Paulo: Casa do Psicólogo.

Nitrini, R., Brucki, S. M. D., Smid, J., Carthery-Goulart, M. T., Anghinah, R., Areza-Fegweres, R., .... Mansur, L. L. (2008). Influence of age, gender and educacional level on performance in the Brief Cognitive Batterry-Edu. Dementia \& Neuropsychologia, 2(2), 114-118.

Nurmi, L., Kettunen, J., Laihosalo, M., Ruuskanen, E. I., Koivisto, A. M., \& Jehkonen, M. (2010). Right hemisphere infarct patients and healthy controls: Evaluation of starting points in cancellation tasks. Journal of the International Neuropsychological Society, 13, 1-8.

Nys, G., Stuart, M., \& Dijkerman, C. (2010) Repetitive exploration towards locations that no longer carry a target in patients with neglect. Journal of Neuropsychology, 4, 33-45.

Ostrosky-Solís, F., Ramírez, M., \& Ardila A. (2004). Effects of Culture and Education on Neuropsychological Testing: A Preliminary Study with Indigenous. Applied Neuropsychology. 11(4), 188-195.

Parente, M. A. M. P., Scherer, L.C., Zimmermann, N., \& Fonseca, R. P. (2009). Evidências do papel da escolaridade na organização cerebral. Revista Neuropsicologia Latinoamericana, 1(1), 72-80.

Plummer, P., Morris, M. E., \& Dunai, J. (2003). Assessment of unilateral neglect. Physical Therapy, 83(8), 732-740.

Rorden, C., \& Karnath, H-O. (2010). A simple measure of neglect severity. Neuropsychologia, 48, 2758-2763.

Rosselli, M., \& Ardila, A. (2003). The impact of culture and education on non-verbal neuropsychological measurements: a critical review. Brain ad Cognition, 52, 326-333.

Rosselli, M., Tappen, R., Williams, C., \& Salvatierra, J. (2006). The relation of education and gender on the attention items of the mini-mental state examination in spanish speaking hispanic elders. Archives of Clinical Neuropsychology, 21(7), 677-686.

Rousseaux, M., Beis, J. M., Pradat-Diehl, P., Martin, Y., Bartolomeo, P., Bernati, T., ... Azouvi, P., (2001). Présentation d'une batterie de dépistage de la negligence spatial: normes et effects de l'âge, du niveau d'éducation, du sexe, de la main et de la latéralité. Revue Neurologique, 157, 1385-1400. doi:10.1136/jnnp.73.2.160

Stern, Y. (2009). Cognitive reserve. Neuropsychologia, 47, 2015-2028.

Talarico, J. N. S., Caramelli, P., Nitrini, R., \& Chaves, E.C. (2007). The influence of schooling on working memory performance in elderly individuals without cognitive decline. Dementia \& Neuropsychologia, 3, 276-281.

Tombaugh, T. N. (2004). Trail Making Test A and B: normative data stratified by age and education. Archives of Clinical Neuropsychology, 19, 203-214.

Vanier, M., Gauthier, L., Lambert, J., Pepin, E. P., Robillard, A., Duboulaz, C. J., ... Joannette, Y. (1990). Evaluation of Left Visuospatial Neglect: norms and discrimination power of two tests. Neuropsychology, 4, 87-96. doi:10.1037/0894-4105.4.2.87

Weintraub, S., \& Mesulam, M.M. (1988). Visual hemispatial inattention: stimulus parameters and exploratory strategies. Journal of Neurology, Neurosurgery and Psychiatry, 51, 1481-1488.

Yassuda, M. S., Diniz, B. S. O., Flaks, M. K., Viola, L. F., Pereira, F. S., Nunes, P. V., \& Forlenza, O. V. (2009). Neuropsychological Profile of Brazilian Older Adults with Heterogeneous Educational Backgrounds. Archives of Clinical Neuropsychology, 24(1), 71-79. doi: 10.1093/arclin/acp009 
Rochele Ferronato Correa da Silva, mestre em Psicologia com ênfase em Cognição Humana pela Pontifícia Universidade Católica do Rio Grande do Sul, e Neuropsicóloga Clínica. Endereço para correspondência: Rua Feijó Júnior, 909, sala 302. Caxias do Sul-RS. CEP 95034-160. Telefone: (54) 9993.4529. E-mail: chelefcs@terra.com.br

Caroline de Oliveira Cardoso, mestranda em Psicologia ênfase em Cognição Humana pela Pontifícia Universidade Católica do Rio Grande do Sul, bolsista do Conselho Nacional de Desenvolvimento Científico e Tecnológico (CNPq), Psicóloga Clínica. E-mail: carolineocardoso@yahoo.com.br Rochele Paz Fonseca, doutora em Psicologia do Desenvolvimento pela Universidade Federal do Rio Grande do Sul, com doutorado sanduíche no Centre de Recherche do Institut Universitaire de Gériatrie de Montréal, Faculdade de Medicina, Universidade de Montreal, e Pós-Doutorado em Clínica e Neurociências - PUC-Rio, em Medicina - UFRJ e em Ciências Biomédicas, na Universidade de Montreal - Canadá, é professora Adjunta da Faculdade de Psicologia e do Programa de Pós-Graduação em Psicologia pela Pontifícia Universidade Católica do Rio Grande do Sul (PUCRS), coordenadora do Grupo de Pesquisa Neuropsicologia Clínica e Experimental (GNCE), Bolsista de Produtividade em pesquisa 2 do Conselho Nacional de Desenvolvimento Científico e Tecnológico (CNPq). E-mail: rochele.fonseca@pucrs.br 